\title{
Hybrid imaging using low-dose, localizing computed tomography enhances lesion localization in renal hyperparathyroidism
}

\author{
Alexander G. Doruyter
}

Divisions of Nuclear Medicine, Tygerberg Hospital, Stellenbosch University, Cape Town, South Africa

\section{Tharbit Hartley}

Divisions of Radiology, Tygerberg Hospital, Stellenbosch University, Cape Town, South Africa

\section{Jonathan W. Ameyo}

Divisions of Nephrology, Tygerberg Hospital, Stellenbosch University, Cape Town, South Africa

\section{Mogamat R. Davids}

Divisions of Nephrology, Tygerberg Hospital, Stellenbosch University, Cape Town, South Africa

\section{James M. Warwick}

Divisions of Nuclear Medicine, Tygerberg Hospital, Stellenbosch University, Cape Town, South Africa

\section{Abstract}

Background: Preoperative localization of parathyroid lesions is potentially beneficial in renal patients with hyperparathyroidism. The aim of this study was to determine the localizing value of hybrid single-photon emission computed tomography combined with low-dose x-ray computed tomography (SPECT/LDCT) compared with SPECT alone and whether the LDCT improved reader confidence.

Patients and methods: A retrospective study examined parathyroid scintigraphy results of patients previously referred with a diagnosis of renal hyperparathyroidism. All patients underwent planar scintigraphy using technetium-99m $\left({ }^{99 \mathrm{~m}} \mathrm{Tc}\right)$-pertechnetate, which was immediately followed with ${ }^{99 m}$ Tc-sestamibi and SPECT/LDCT $\sim 60 \mathrm{~min}$ after sestamibi injection and a delayed static image to assess differential washout. Planar subtraction images were generated. Two nuclear physicians, assisted by a radiologist, reported on planar+SPECT images followed by planar+SPECT/LDCT images.

Results: Thirty-seven patients (males: 21 ; females: 16$)$ were included (mean age 39 years, range: 23.9-55.5). Mean creatinine level was $878 \mu \mathrm{mol} / \mathrm{l}$ (109-1839), mean corrected calcium level was 2.42 $\mathrm{mmol} / \mathrm{l}$ (1.77-3.64), and median parathyroid hormone level was $156.2 \mathrm{pmol} / \mathrm{l}(2.4$ to >201). Twentythree patients had positive planar and SPECT results (46 lesions), six had positive planar results only, and eight had negative scintigraphy results. In the patients with positive planar and SPECT results, 35 lesions were classified as eutopic and 11 as ectopic. After the addition of LDCT, localization of five eutopic lesions (on SPECT) was amended to ectopic locations (on SPECT/LDCT), principally by 
identifying posteriorly situated glands. The addition of LDCT led to increased confidence in localization in all cases.

Conclusion: Compared with SPECT alone, SPECT/LDCT is beneficial in preoperative localization of lesions in renal hyperparathyroidism.

Keywords: parathyroid scintigraphy; preoperative localization; renal hyperparathyroidism; singlephoton emission computed tomography/computed tomography

\section{Introduction}

In contrast to de-novo neoplasia or hyperplasia of one or more of the parathyroid glands in primary hyperparathyroidism, secondary hyperparathyroidism refers to parathyroid hyperplasia in response to a chronic hypocalcaemic condition (most commonly renal failure), and tertiary hyperparathyroidism refers to hyperplastic parathyroid glands developing functional autonomy, which is no longer suppressible by correction of hypocalcaemia.

Parathyroid scintigraphy has an established role in the detection of hyperfunctioning parathyroid tissue when surgery is being considered $\underline{1}$. Scintigraphy's main role in renal hyperparathyroidism is the detection of residual or recurrent hyperfunctioning glands after surgery $\underline{1-9}$. Routine preoperative scintigraphy in this patient group remains controversial. Those opposing its use cite the poor sensitivity and localization accuracy of the technique in multiglandular disease $\underline{5,10,11}$. In addition, renal hyperparathyroidism typically involves multiple glands and requires bilateral surgical exploration regardless of preoperative lesion localization. This raises questions about the value of scintigraphy to shorten and/or simplify surgery. Routine preoperative scintigraphy is, however, performed by some centres to reduce operative time and the extent of dissection.

Reducing operative time is particularly important as these patients typically carry a high anaesthetic risk related to underlying medical conditions causing renal dysfunction or renal disease itself $\underline{12}$. In addition, the detection of supernumerary and ectopic glands is expected to reduce the rate of surgical failure 5,13-17, which, interestingly, is higher in renal hyperparathyroidism $\underline{18-21}$. There is no published literature examining the clinical impact of preoperative localization in renal hyperparathyroidism.

It is expected that the higher the number of lesions detected by scintigraphy (greater sensitivity), and the more accurately these lesions are localized and identified as eutopic or ectopic, the more likely it is that preoperative scintigraphy will contribute to the management of renal hyperparathyroidism.

There is evidence that hybrid imaging using single-photon emission computed tomography (SPECT) combined with low-dose x-ray computed tomography (LDCT) has no value in improving the sensitivity of scintigraphy compared with SPECT alone. This is true of both primary disease and renal hyperparathyroidism $\underline{8,22,23}$. There is, however, indirect evidence to suggest that LDCT improves localization in preoperative scintigraphy for renal hyperparathyroidism. First, more accurate localization was found with SPECT/LDCT in studies that mainly examined patients with primary hyperparathyroidism $\underline{8,23-26}$; second, more accurate localization has been achieved using SPECT fused with diagnostic-quality computed tomography (CT) in renal hyperparathyroidism 27. 
Our institution performs SPECT/LDCT for all cases of parathyroid scintigraphy, including renal hyperparathyroidism. The aim of this study was to determine the impact of the LDCT component on lesion localization, given the additional imaging time and radiation dose involved. We hypothesized that the additional LDCT component would improve lesion localization and improve reader confidence of lesion localization. To the authors' knowledge, no study has directly examined whether SPECT/LDCT has any impact in terms of lesion localization in preoperative scintigraphy of renal hyperparathyroidism when compared with SPECT alone.

\section{Methods}

\section{Patient selection}

All parathyroid studies of patients with renal hyperparathyroidism referred to our department between February 2008 and June 2011 were reviewed retrospectively. In patients with repeat scans, a single study with the chronologically closest biochemical data was used. The study was conducted in accordance with established ethical guidelines after approval by the Health Research Ethics Committee of Stellenbosch University (protocol no. N11/06/197).

\section{Image acquisition}

Both subtraction and dual-phase techniques were performed using Elscint APEX SPX 4 or Elscint APEX SP 4 gamma cameras (General Electric Medical Systems, Milwaukee, Wisconsin, USA). Imaging was performed using a 3-mm pinhole collimator, with a zoom factor of 1.8 and a $256 \times 256$ acquisition matrix. Fifteen minutes after intravenous injection of 74-111 MBq of technetium-99m $\left({ }^{99 \mathrm{~m}} \mathrm{Tc}\right)$-pertechnetate, two anterior images were obtained over the thyroid (with and without a marker over the sternal notch) at a distance of $5 \mathrm{~cm}$ from the collimator to the surface of the neck for $300 \mathrm{~s}$ each. Without allowing the patient to move, $740 \mathrm{MBq}$ of ${ }^{99 \mathrm{~m}} \mathrm{Tc}-$-sestamibi was then injected through an existing intravenous line, and 5 min later five consecutive planar images were obtained in the same way. Planar subtraction images were later generated from the acquired sestamibi and pertechnetate planar images.

A single anterior image with a wider field of view was then obtained at a distance of $20 \mathrm{~cm}$ for $120 \mathrm{~s}$. A delayed pinhole image was acquired after 2-3 $\mathrm{h}$ using the same imaging parameters as the early images to assess differential washout. Planar subtraction images were generated using HERMES MultiModality Software (Hermes Medical Solutions, Stockholm, Sweden).

SPECT/LDCT imaging was performed 60 min after injection of sestamibi, with Infinia Hawkeye (General Electric Medical Systems). Projection data were acquired using a low-energy high-resolution collimator with a zoom factor of 1 , a matrix size of $128 \times 128$ and a step and shoot protocol of $30 \mathrm{~s} / \mathrm{step}$ and $3^{\circ}$ per step over 360 degrees. This was immediately followed by a low-dose single-slice CT using a slice thickness of $10 \mathrm{~mm}$, current of $2.5 \mathrm{~mA}$, voltage of $140 \mathrm{kV}$, and a 256×256 matrix. The CT was acquired at two revolutions/min for a total imaging time of 10-12 min. The CT was reconstructed using a Hann filter with a cutoff frequency of 1 cycle/cm.

Transverse SPECT images were reconstructed using an ordered subset expectation maximization algorithm with 16 iterations and 30 subsets, incorporating collimator blurring, scatter and attenuation 
correction. Nonuniform attenuation correction was performed using the CT data. A Butterworth postprocessing filter with 1.4 cycles/cm cutoff frequency was applied to the SPECT data.

\section{Image interpretation}

Two nuclear medicine physicians and one radiologist, who were blinded to the biochemical, surgical and pathological results of the patients, interpreted the scintigraphic studies twice. They first reported the pinhole images and SPECT, and then SPECT/LDCT results. The reporters gave a consensus interpretation of the presence of hyperfunctioning parathyroid tissue and its location (eutopic: left/right, superior/inferior; ectopic: posterior, inferior, intrathyroidal, mediastinal). In addition, readers indicated whether or not the LDCT improved their confidence in lesion localization. Viewing of both planar images as well as SPECT and LDCT studies (including image fusion) was performed on a DELL Optiplex GX745 workstation (Dell Inc., Round Rock, Texas, USA) using programs from the HERMES GOLD application suite v2.11 (HERMES Display and Scale and Volume Display Software; Hermes Medical Solutions, Stockholm, Sweden).

\section{Measures of renal disease}

Information on the underlying renal pathology, current medical management of hyperparathyroidism, dialysis treatment and whether a prior renal transplant had been performed was extracted from patient records and recorded using a standardized data sheet.

\section{Biochemical and pathological markers}

Serum levels of parathyroid hormone (PTH) closest to the time of the scan were obtained, together with recently corrected calcium, phosphate and creatinine measurements. When surgery was performed, the pathology and location of resected tissue as well as postsurgical PTH levels were recorded when available.

\section{Results}

\section{Demographics}

Of the 41 scans of patients with secondary or tertiary hyperparathyroidism eligible for inclusion, four scans were excluded (three scans were repeats in the same patients, whereas a fourth patient had a possible history of primary hyperparathyroidism). Scans from the remaining 37 patients were included in the study. Twenty-one male patients and 16 female patients were included with a mean age of 39 years (range: $23.9-55.5$ years).

\section{Imaging findings}

The impact of LDCT on imaging findings is summarized in Table 1. 


\section{Detection}

Forty-six lesions were visible on SPECT (and planar images) in 23 patients. Among these patients, nine had a single lesion on SPECT imaging, six had two lesions, seven had three lesions and one patient had four lesions. Of the 14 patients with negative SPECT, six patients had lesions visible on pinhole imaging and eight patients had negative results on both pinhole imaging and SPECT. These findings did not change with the addition of LDCT.

\section{Localization}

Of the 23 patients with lesions seen on SPECT, 15 patients had 27 lesions, all of which were thought to be eutopic. The remaining eight patients had 19 lesions, of which one lesion was thought to be ectopic in each of five patients and two were thought to be ectopic in each of three patients. Thus, of the 46 lesions identified on SPECT, 35 were interpreted as eutopic and 11 as ectopic.

The addition of LDCT resulted in changes to lesion localization in five patients. All five, thought to have only eutopic lesions on SPECT alone, had a single lesion reclassified as ectopic. This resulted in 30 eutopic lesions and 16 ectopic lesions. Lesions for which localization changed with the addition of LDCT (as well as details of the localization change) have been tabulated (Table 1). An example of localization change is provided in Fig. 1. The use of hybrid imaging improved the reader confidence in lesion localization in all cases.

\section{Incidental findings}

With the benefit of LDCT, incidental foci of sestamibi uptake were localized to vertebrae in two patients.

\section{Pathology and management measures}

All the patients included in our study had chronic kidney disease. The majority of patients were on either haemodialysis $(n=25)$ or peritoneal dialysis $(n=9)$. Only two patients were not on dialysis, and in one patient this information was not available. Twenty-nine patients were receiving calcium supplementation at the time of scanning, whereas five patients were not on calcium supplementation; this information was not available in three patients. Similarly, 24 patients were on vitamin D supplementation, whereas 10 patients were not on such supplementation; this information was not available for three patients. Fourteen patients had a history of a renal transplantation.

Biochemistry results are summarized in Table 2. As the upper detectable limit of PTH at our institution is $201 \mathrm{pmol} / \mathrm{l}$, and 11 patients exceeded this level, an accurate mean and maximum for PTH could not be determined (a median was therefore used). The PTH level was raised in all patients except one, in whom the level was inappropriately normal in the context of a raised (corrected) calcium level. An alternative explanation was that this normal value represented a hook effect, which was not tested for. The mean time intervals (range) between biochemistry results and scintigraphy were as follows: PTH 1.8 months (0.1-4.6 months); corrected calcium and phosphate -1.7 months ( $0-4.5$ months); creatinine -1.7 months $(0-5.1$ months $)$. 
Of the 37 patients included in the study, 11 subsequently underwent parathyroid surgery. Records were available for 10 of these patients. In this subgroup, 38 lesions (histologically demonstrating parathyroid hyperplasia) were resected, whereas only 12 were detected on scintigraphy (identification rate $32 \%$ ). Unfortunately, operative notes did not accurately record the location of resected glands. The mean mass of resected parathyroid glands was $0.91 \mathrm{~g}$ with values ranging from 0.1 to $3.62 \mathrm{~g}$ (normal $<0.04 \mathrm{~g}$ ). Three resected parathyroid glands (in one patient) were not weighed because of a laboratory oversight.

PTH levels after surgery were available in nine patients. These demonstrated normalization in seven, near normalization in one and persistent elevation in one. The mean time interval between surgery and repeat PTH was 3.2 months (range: 0.7-8.7 months).

\section{Discussion}

In this study we found that the addition of LDCT changed the location of lesions from eutopic to ectopic in five out of 37 patients and improved reader confidence in lesion location in all positive studies. The hybrid modality also allowed the localization of other incidental pathologies in two patients. To our knowledge this is the first study to specifically look at the role of SPECT/LDCT in renal hyperparathyroidism.

The changed localization of five lesions in five patients with the addition of LDCT in our study is consistent with the results of other studies that compared SPECT with SPECT/LDCT.

Gayed et al. 22, in their study on 48 patients with primary hyperparathyroidism, compared SPECT and SPECT/LDCT. Reader 1 reported 40 scans as positive and reader 2 reported 38 scans as positive. The addition of LDCT assisted in localization in four lesions in four out of 48 patients $\underline{22}$. Öksüz et al. $\underline{23}$ assessed preoperative SPECT/LDCT in 35 patients with mostly primary hyperparathyroidism. The investigators reported that SPECT/LDCT provided superior topographic information, particularly in patients with ectopic adenomas, although the number of lesions in which LDCT changed localization was not reported $\underline{23}$.

It is possible that SPECT/LDCT may make a greater contribution in patients with a multinodular goitre. Serra et al. $\underline{8}$ compared SPECT and SPECT/LDCT in 16 patients with hyperparathyroidism, of whom 12 had a multinodular goitre. In their study, LDCT changed localization in a large proportion of patients - seven out of 14 lesions in six out of 10 patients in the primary group and seven out of nine lesions in five out of six patients in the secondary group $\underline{8}$. In 33 patients with concomitant primary hyperparathyroidism and multinodular goitre, Pata et al. 25 reported that, when SPECT was compared with SPECT/LDCT in different groups, SPECT/LDCT was found to be more accurate in lateralizing parathyroid lesions. Change in localization of individual lesions was not studied 25.

Of the five lesions in which LDCT resulted in changed localization in our study, three were reclassified as being posteriorly situated. This is consistent with previous findings demonstrating that LDCT improves the detection of posteriorly situated glands. In the study by Serra et al. $\underline{8}$, in three patients (all with primary disease) additional LDCT identified a posteriorly situated (retrotracheal) gland. Lavely et al. $\underline{24}$, in their study on patients with primary hyperparathyroidism, reported that the major benefit of the hybrid modality (incorporating LDCT) was its ability to distinguish inferior from inferoposterior glands (situated in the tracheo-oesophageal groove). 
Not all studies have reported a benefit with LDCT. In a retrospective comparison between the accuracy of localization of SPECT $(n=165)$ and that of SPECT/LDCT $(n=131)$ in primary hyperparathyroidism, the authors found no difference in the accuracy of lesion localization between the two groups. This result may, however, be related to the two modalities being compared in different patients $\underline{28}$. Similarly, Gayed et al. 22 reported that SPECT/CT did not detect more ectopic lesions compared with SPECT alone in a study of patients with primary disease.

In this study, nuclear medicine physicians were assisted by a radiologist with good knowledge of variations in parathyroid anatomy. It is worth noting that without sound anatomical knowledge the impact of LDCT may not be fully realized.

Reader confidence in localization improved in all our cases with the addition of LDCT. This is consistent with a report by Jacene et al. $\underline{29}$ on the advantages of hybrid imaging with SPECT/LDCT over SPECT alone in which the authors stated, 'functional images often lack the anatomic detail necessary for accurate lesion localisation, which can decrease reader confidence in image interpretation and lead to uncertain or erroneous interpretations'.

Very little research has been directed towards the clinical impact of LDCT in preoperative imaging of hyperparathyroidism. In a study by Serra et al. $\underline{8}$, hybrid imaging resulted in a modification of the surgical approach in three out of 10 patients with primary disease when lesions were determined to be posteriorly situated. Lavely et al. 24, commenting on the ability of SPECT/LDCT to distinguish between inferior and inferior-posterior localizations, pointed out that surgery for a gland in the inferior-posterior location is frequently more complex because of its intimate association with the recurrent laryngeal nerve and that preoperative knowledge of this ectopic location facilitates surgical exploration.

In a study by Pata et al. $\underline{25}$ in patients with concomitant primary hyperparathyroidism and multinodular goitre, the mean operative time was less in the SPECT/LDCT group compared with the SPECT group. In addition, at least two studies in primary hyperparathyroidism mention reductions in operation time, or facilitation of resection with the use of SPECT/LDCT, although neither of these studies directly compared SPECT and SPECT/LDCT in terms of a surgical or clinical outcome measure $\underline{30,31}$.

The lesions localized to vertebrae in two patients on LDCT represent potentially important incidental findings. The differential diagnosis of these lesions includes brown tumours and metastatic lesions. No subsequent record of malignancy was found in the medical records of these patients and these lesions were hypothesized to represent brown tumours.

Literature on the impact of LDCT on parathyroid scintigraphy in terms of incidental findings is limited. Treglia et al. 32 describe a case of primary hyperparathyroidism in which SPECT/LDCT localized a lesion with sestamibi uptake to the third left rib, which was subsequently demonstrated to be a brown tumour. Brown tumours may occur in renal hyperparathyroidism and are known to take up sestamibi $\underline{33-38}$. Vertebral brown tumours are reportedly rare $\underline{39}$.

A limitation of this retrospective study was that no information was available on the clinical impact of the scintigraphy, or on the findings during surgery. It is assumed that SPECT/LDCT is more accurate when its findings are discordant with those of SPECT. Previously, however, hybrid imaging has been found to be $100 \%$ accurate in lesion localization when correlated with surgical findings $\underline{8}$. 
By virtue of its improved references to anatomical landmarks, it is probable that any differences in localization between SPECT and SPECT/LDCT in our group would reflect a deficiency of SPECT in terms of localization.

Similarly, although LDCT changed lesion localization in five out of 37 patients, there are no measures of the impact of the information on patient management (e.g. changed surgical approach, changed anaesthetic plan).

An additional limitation is that SPECT was commenced $1 \mathrm{~h}$ after MIBI injection, which is relatively late. This is likely to account for six patients with lesions detected on planar imaging but not on SPECT 24. It is possible that the impact of SPECT/LDCT may have been greater if SPECT had been performed earlier. Similarly, the identification rate of $32 \%$ would probably have been higher with earlier SPECT.

Finally, this study was limited to a single consensus report by a group of three doctors. As such, it provides no measures of the inter-reporter or intra-reporter variability of findings.

\section{Conclusion}

In patients with renal hyperparathyroidism, the addition of LDCT results in better localization of hyperfunctioning parathyroid tissue and improved reader confidence. These improvements are likely to have a significant impact on further patient management.

\section{Acknowledgements}

The authors thank Prof. Martin Kidd, Centre for Statistical Consultation, Department of Statistics and Actuarial Sciences, Stellenbosch University and Dr Mariza Hoffmann, Division of Chemical Pathology, National Health Laboratory Service, Tygerberg Hospital, Stellenbosch University.

\section{Conflicts of interest}

There are no conflicts of interest. 


\section{Tables}

Table 1

Table 1 Lesions in which addition of low-dose x-ray computed tomography changed localization

\begin{tabular}{lccl}
\hline Patient & Lesion & $\begin{array}{c}\text { Location: } \\
\text { planar+SPECT }\end{array}$ & Location: planar+SPECT/LDCT \\
\hline 15 & 17 & Eutopic: LI & Ectopic: posterior to L SC joint \\
21 & 28 & Eutopic: LI & Ectopic: LIP \\
24 & 33 & Eutopic: LI & Ectopic: inferior midline \\
29 & 38 & Eutopic: LI & Ectopic: LIP \\
37 & 45 & Eutopic: LS & Ectopic: LSP
\end{tabular}

L, left; LDCT, low-dose x-ray computed tomography; LI: left inferior; LIP, left inferior-posterior; LS, left superior; LSP, left superior-posterior; RI, right inferior; $\mathrm{RS}$, right superior; SC, sternoclavicular.

Table 2

Table 2 Biochemistry results of study group

\begin{tabular}{lccc}
\hline Biochemical parameters & Mean & Range & Reference range and units \\
\hline $\begin{array}{l}\text { Parathyroid hormone } \\
\quad \text { (PTH) }\end{array}$ & $156.2^{\mathrm{a}}$ & 2.4 to $>201$ & $1.2-8.5 \mathrm{pmol} / \mathrm{l}$ \\
Corrected calcium & 2.42 & $1.77-3.64$ & $2.05-2.56 \mathrm{mmol} / \mathrm{l}$ \\
Phosphate & 1.85 & $0.74-2.95$ & $0.8-1.4 \mathrm{mmol} / \mathrm{l}$ \\
Creatinine & 878 & $109-1839$ & $60-120 \mu \mathrm{mol} / \mathrm{l}$ \\
\hline Median. & & &
\end{tabular}




\section{Figures}

Figure 1

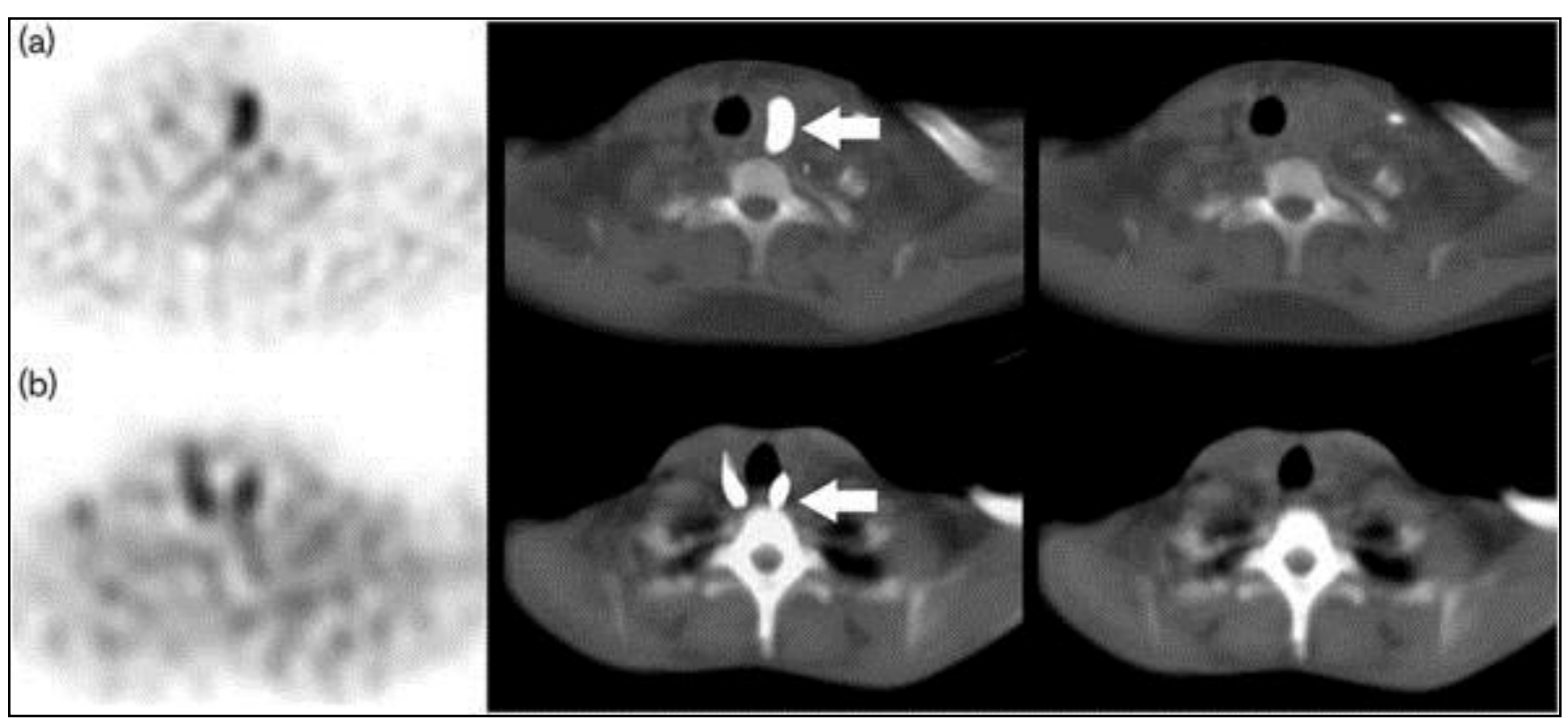

Fig. 1. Example of the ability of LDCT to distinguish between eutopic gland (a) and posteriorly-situated gland (b) indicated by solid arrows. Transverse images from left to right: SPECT, fused (windowing and background adjusted) SPECT/LDCT, and LDCT. LDCT, low-dose x-ray computed tomography; SPECT, single-photon emission computed tomography. 


\section{References}

1. Hindié E, Ugur O, Fuster D, O’Doherty M, Grassetto G, Ureña P, et al..2009 EANM parathyroid guidelines. Eur J Nucl Med Mol Imaging2009;36:1201-1216.

2. Greenspan BS, Dillehay G, Intenzo C, Lavely WC, O’Doherty M, Palestro CJ, et al.. SNM Practice Guideline for Parathyroid Scintigraphy 4.0. J Nucl Med Technol2012;40:111-118.

3. Mitchell BK, Merrell RC, Kinder BK. Localization studies in patients with hyperparathyroidism. Surg Clin North Am1995;75:483-498.

4. Neumann DR, Esselstyn CB Jr, Madera AM. Sestamibi/iodine subtraction single photon emission computed tomography in reoperative secondary hyperparathyroidism. Surgery2000;128:22-28.

5. Lai ECH, Ching ASC, Leong HT. Secondary and tertiary hyperparathyroidism: role of preoperative localization. ANZ J Surg2007;77:880-882.

6. Chesser AM, Carroll MC, Lightowler C, Macdougall IC, Britton KE, Baker LR. Technetium99m methoxy isobutyl isonitrile (MIBI) imaging of the parathyroid glands in patients with renal failure.Nephrol Dial Transplant1997;12:97-100.

7. Gasparri G, Camandona M, Bertoldo U, Sargiotto A, Papotti M, Raggio E, et al.. The usefulness of preoperative dual-phase ${ }^{99 \mathrm{~m}} \mathrm{Tc}$ MIBI-scintigraphy and IO-PTH assay in the treatment of secondary and tertiary hyperparathyroidism. Ann Surg2009;250:868-871.

8. Serra A, Bolasco P, Satta L, Nicolosi A, Uccheddu A, Piga M. Role of SPECT/CT in the preoperative assessment of hyperparathyroid patients. Radiol Med2006;111:999-1008.

9. Papathanassiou D, Flament JB, Pochart JM, Patey M, Marty H, Liehn JC, et al.. SPECT/CT in localization of parathyroid adenoma or hyperplasia in patients with previous neck surgery. Clin Nucl Med2008;33:394-397.

10. Pons F, Torregrosa JV, Vidal-Sicart S, Sabater L, Fuster D, Fernández-Cruz L, et al.. Preoperative parathyroid gland localization with technetium-99m sestamibi in secondary hyperparathyroidism. Eur J Nucl Med1997;24:1494-1498.

11. Pham TH, Sterioff S, Mullan BP, Wiseman GA, Sebo TJ, Grant CS. Sensitivity and utility of parathyroid scintigraphy in patients with primary versus secondary and tertiary hyperparathyroidism. World J Surg2006;30:327-332

12. Rang ST, West NL, Howard J, Cousins J. Anaesthesia for chronic renal disease and renal transplantation. EAU-EBU Update Ser2006;4:246-256.

13. Lomonte C, Buonvino N, Selvaggiolo M, Dassira M, Grasso G, Vernaglione L, et al.. Sestamibi scintigraphy, topography, and histopathology of parathyroid glands in secondary hyperparathyroidism. Am J Kidney Dis2006;48:638-644.

14. Sfakianakis GN, Irvin GL III, Foss J, Mallin W, Georgiou M, Deriso GT, et al.. Efficient parathyroidectomy guided by SPECT-MIBI and hormonal measurements. J Nucl Med1996;37:798-804.

15. Périé S, Fessi H, Tassart M, Younsi N, Poli I, St Guily JL, et al.. Usefulness of combination of high-resolution ultrasonography and dual-phase dual-isotope iodine 123/technetium Tc 99m sestamibi scintigraphy for the preoperative localization of hyperplastic parathyroid glands in renal hyperparathyroidism. Am J Kidney Dis2005;45:344-352.

16. Wheeler M. Preoperative parathyroid scanning in secondary hyperparathyroidism. Lancet1999;353:2174-2175.

17. Loftus KA, Anderson S, Mulloy AL, Terris DJ. Value of sestamibi scans in tertiary hyperparathyroidism. Laryngoscope2007;117:2135-2138. 
18. Neonakis E, Wheeler MH, Krishnan H, Coles GA, Davies F, Woodhead JS. Results of surgical treatment of renal hyperparathyroidism. Arch Surg1995;130:643-648.

19. Gasparri G, Camandona M, Abbona GC, Papotti M, Jeantet A, Radice E, et al.. Secondary and tertiary hyperparathyroidism: causes of recurrent disease after 446 parathyroidectomies. Ann Surg2001;233:65-69.

20. Rothmund M, Wagner PK. Reoperations for persistent and recurrent secondary hyperparathyroidism. Ann Surg1988;207:310-314.

21. Tominaga Y, Numano M, Tanaka Y, Uchida K, Takagi H.Surgical treatment of renal hyperparathyroidism. Semin Surg Oncol1997;13:87-96Available at: http://onlinelibrary.wiley.com/doi/10.1002/(SICI) 1098-2388(199703/04)13:2\%3C87::AID-SSU4\%3E3.0.CO;2Y/abstract. [Accessed 12 September 2013].

22. Gayed IW, Kim EE, Broussard WF, Evans D, Lee J, Broemeling LD, et al.. The value of ${ }^{99} \mathrm{~m}$ Tc-sestamibi SPECT/CT over conventional SPECT in the evaluation of parathyroid adenomas or hyperplasia. J Nucl Med2005;46:248-252.

23. Oksüz MO, Dittmann H, Wicke C, Müssig K, Bares R, Pfannenberg C, et al.. Accuracy of parathyroid imaging: a comparison of planar scintigraphy, SPECT, SPECT-CT, and C-11 methionine PET for the detection of parathyroid adenomas and glandular hyperplasia. Diagn Interv Radiol2011;17:297-307.

24. Lavely WC, Goetze S, Friedman KP, Leal JP, Zhang Z, Garret-Mayer E, et al.. Comparison of SPECT/CT, SPECT, and planar imaging with single- and dual-phase (99m)Tc-sestamibi parathyroid scintigraphy. J Nucl Med2007;48:1084-1089.

25. Pata G, Casella C, Besuzio S, Mittempergher F, Salerni B. Clinical appraisal of 99m technetium-sestamibi SPECT/CT compared to conventional SPECT in patients with primary hyperparathyroidism and concomitant nodular goiter. Thyroid2010;20:1121-1127.

26. Ruf J, Seehofer D, Denecke T, Stelter L, Rayes N, Felix R, et al.. Impact of image fusion and attenuation correction by SPECT-CT on the scintigraphic detection of parathyroid adenomas.Nuklearmedizin2007;46:15-21.

27. Wimmer G, Profanter C, Kovacs P, Sieb M, Gabriel M, Putzer D, et al.. CT-MIBI-SPECT image fusion predicts multiglandular disease in hyperparathyroidism. Langenbecks Arch Surg2010;395:73-80.

28. Sharma J, Mazzaglia P, Milas M, Berber E, Schuster DM, Halkar R, et al.. Radionuclide imaging for hyperparathyroidism (HPT): Which is the best technetium-99m sestamibi modality? Surgery2006;140:856-865.

29. Jacene HA, Goetze S, Patel H, Wahl RL, Ziessman HA. Advantages of hybrid SPECT/CT vs SPECT alone. Open Med Imaging J2008;2:67-79.

30. Harris L, Yoo J, Driedger A, Fung K, Franklin J, Gray D, et al.. Accuracy of technetium-99m SPECT-CT hybrid images in predicting the precise intraoperative anatomical location of parathyroid adenomas. Head Neck2008;30:509-517.

31. Krausz Y, Bettman L, Guralnik L, Yosilevsky G, Keidar Z, Bar-Shalom R, et al.. Technetium99m-MIBI SPECT/CT in primary hyperparathyroidism. World J Surg2006;30:76-83.

32. Treglia G, Dambra DP, Bruno I, Mulè A, Giordano A. Costal brown tumor detected by dualphase parathyroid imaging and SPECT-CT in primary hyperparathyroidism. Clin Nucl Med2008;33:193-195.

33. Meng Z, Zhu M, He Q, Tian W, Zhang Y, Jia Q, et al.. Clinical implications of brown tumor uptake in whole-body ${ }^{99 \mathrm{~m}} \mathrm{Tc}$-sestamibi scans for primary hyperparathyroidism. Nucl Med Commun2011;32:708-715. 
34. Ohnishi H, Abe M, Miyagawa M, Nishimura K. Tl-201, Tc-99m sestamibi, and Tc-99m HMDP uptake in multiple brown tumors. Clin Nucl Med2003;28:601-603.

35. Pai M, Park CH, Kim BS, Chung YS, Park HB. Multiple brown tumors in parathyroid carcinoma mimicking metastatic bone disease. Clin Nucl Med1997;22:691-694.

36. Dinauer PA, Balingit AG, Rivera JE. Tc-99m sestamibi imaging of brown tumors of primary hyperparathyroidism. Clin Nucl Med1996;21:192-196.

37. Rubin MR, LiVolsi VA, Bandeira F, Caldas G, Bilezikian JP. Tc99m-sestamibi uptake in osteitis fibrosa cystica simulating metastatic bone disease. J Clin Endocrinol Metab2001;86:5138-5141.

38. Lu G, Shih WJ, Xiu JY. Technetium-99m MIBI uptake in recurrent parathyroid carcinoma and brown tumors. J Nucl Med1995;36:811-813.

39. Fineman I, Johnson JP, Di-Patre PL, Sandhu H. Chronic renal failure causing brown tumors and myelopathy. Case report and review of pathophysiology and treatment. J Neurosurg1999;90:242-246. 